\title{
Terrestrial mammals of the satellite islands of Sardinia (Italy)
}

\author{
Marco Masseti \\ Department of Biology of the University of Florence, Via del Proconsolo 12, 50122 Firenze, Italy
}

\begin{abstract}
The mammalian fauna of the satellite islands and islets of Sardinia (Italy) is still imperfectly known. Only few data are available for some of them, while several others are still almost regarded as terra incognita. Complete information on the extant non-volant terrestrial mammals is available only for Asinara, Tavolara, and Molara, whereas historical and present data are available on the mammals of San Pietro. Further information on the non-flying taxa occasionally appears in specialised literature. At present, 15 species occur in the circum-Sardinian archipelagos. Only 6 of them, however, have been reported from the largest island, San Pietro, whereas the smaller Asinara hots at least 13 species, excluding local domestic breed such as the dwarf donkey. Data on the distribution of chiropters have been provided by a few studies carried out over time. Of the 21 species of bats found in Sardinia, at least 11 were recorded from the small islands.
\end{abstract}

KEY WORDS Non-volant terrestrial mammals; bats.

Received 13.06.2018; accepted 05.10.2018; published online 20.12.2019

Proceedings of the 4th International Congress on Biodiversity "Man, Natural Habitats and Euro-Mediterranean Biodiversity", November 17th-19th, 2017 - Malta

\section{INTRODUCTION}

The mammalian fauna of the satellite islands and islets of Sardinia (Italy) is still imperfectly known.

Only few data are available for some species, while several others are still almost regarded as "terra incognita". Complete information on the extant non-volant terrestrial mammals is available only for Asinara (Torre \& Monbailliu, 1993; Cossu et al., 1994), Tavolara (Ranzi, 1971; Trainito, 2008), and Molara (Sposimo et al., 2012), whereas Zava et al. (1995) make available historical and present data on the mammals of San Pietro.

Further information on the non-flying taxa occasionally appears in specialised literature.

\section{DISCUSSION}

At present, 15 species occur in the circum-Sardinian archipelagos. Only 6 of them, however, have been reported from the largest island, San Pietro, whereas the smaller Asinara hots at least 13 species, excluding the local dwarf donkey domestic breed. The only species which appears to be more widespread is the black rat, Rattus rattus (Linnaeus, 1758), occurring almost everywhere, a part from the islets of Spargiotto, Barrettini, and Foradada (Capo Caccia) (Martin et al., 2000).

The brown rat, Rattus norvegicus (Berkenhout, 1769), has been instead reported only from Asinara (Sarà, 1998) and Tavolara (Capizzi \& Santini, 1999e, 2002e; Trainito, 2008, 2011). Recent surveys, however, did not confirm the occurrence of 
this species on the latter island (Paolo Sposimo, 2014 pers. com.). The garden dormouse, Eliomys quercinus (Linnaeus, 1766) (Fig. 1), the wood mouse, Apodemus sylvaticus (Linnaeus, 1758), and the weasel, Mustela nivalis Linnaeus, 1766 (Fig. 2), are present on Asinara.

Another carnivore, the fox, Vulpes vulpes (Linnaeus, 1758), has been reported from Isola dei Cavoli where it was introduced after 1995 to prevent the damages produced by the rats, although its presence was defined by Scrugli \& Cogoni (1995) "as only occasionally verifiable". Observations of this carnivore are also available for San Pietro (Zava et al., 1995). The western European hedgehog, Erinaceus europaeus Linnaeus, 1758, occurs on Asinara (Torre \& Monbailliu, 1993; Cossu et al., 1994; Amori \& Masseti, 1996), San Pietro (Zava et al., 1996), and Caprera (Cossu et al., 1994). The North African white-toothed shrew or Pantellerian shrew, Crocidura pachyura (Küster, 1835) inhabits Asinara (Amori \& Masseti, 1996; Torre \& Monbailliu, 1993), and Caprera (Amori \& Masseti, 1996; Thibault et al., 1988), whereas the pygmy whitetoothed shrew, Suncus etruscus (Savi, 1822) has been only certainly reported from Asinara (Torre \& Monbailliu, 1993; Amori \& Masseti, 1996; Contoli $\&$ Amori, 2008). On Tavolara, data on the presence of the latter species are only known from the first half of 1970s (Ranzi, 1971; Pratesi \& Tassi, 1973). Together with the house mouse, Mus musculus Linnaeus, 1758, dispersed on Asinara (Torre \& Monbailliu, 1993; Amori \& Masseti, 1996; Capizzi \& Santini, 1999c, 2002c), Tavolara ( Baccetti et al.,

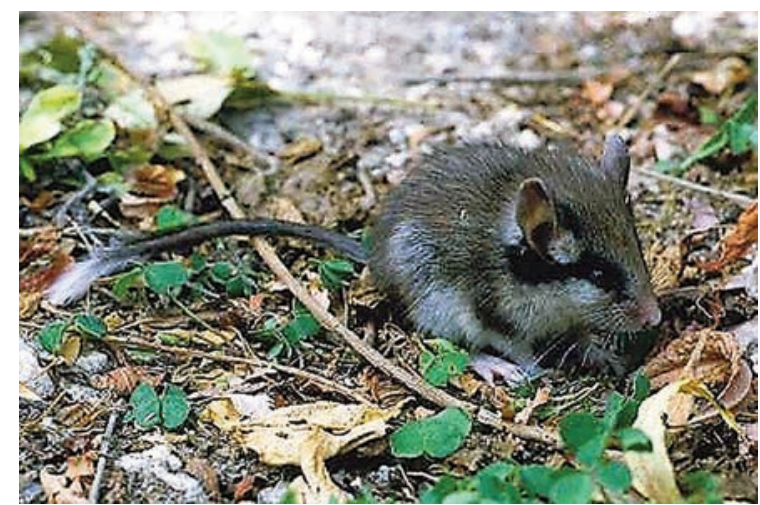

Figure 1. The garden dormouse, Eliomys quercinus (Linnaeus, 1766), is among the non-volant mammals present on Asinara (photo by Roberto Meloni).
2009; Sposimo et al., 2012; Ragionieri et al., 2013), Isola Piana, Isola dei Cavalli, Proratora, Reulino or Isolotto rosso (present work), the rabbit, Oryctolagus cuniculus (Linnaeus, 1758), is one of the most widespread species and occurs on Isola Piana (off Tavolara) (Trainito, 2008; Trainito \& Navone, 2011), San Pietro (Cetti, 1774; Vallebona, 1974; Racheli, 1981; Zava et al., 1996), Caprera, Santa Maria, Razzoli, Spargiotto, Spargi, Mal di Ventre, Vacca, Toro, and Isola Rossa (Spagnesi, 1999b and 2002b). Pratesi \& Tassi $(1971,1973)$ noted that the latter species was also reported from Tavolara, but it is no more present. According instead to Segala (1991) and Spagnesi (1999a and 2002a), the brown hare, Lepus europaeus Pallas, 1778, occurs on Asinara; Spagnesi (1999a, 2002a) reported the occurrence of this species from Maddalena and San Pietro, too.

The situation of the ungulates is rather different and only three species of this taxonomic group are presently known for the satellite islands of Sardinia. Ancient populations of wild goats, Capra aegagrus Erxleben, 1777, are traditionally reported from Asinara (Toschi, 1953; De Beaux, 1955; Couturier, 1959; Massoli Novelli, 2003; Masseti, 2008, 2009, 2014) (Fig. 3) and Tavolara (Cetti, 1774; Valery, 1837; McGrigor, 1866; Toschi, 1953; Couturier, 1959; Masseti, 2008, 2009, 2014). Nowadays, their occurrence on both islands appears seriously compromised, and no individual of the original population still remains on Tavolara, where domestic goats were introduced by man. Most of the human population of Tavolara was displaced in 1962 when a

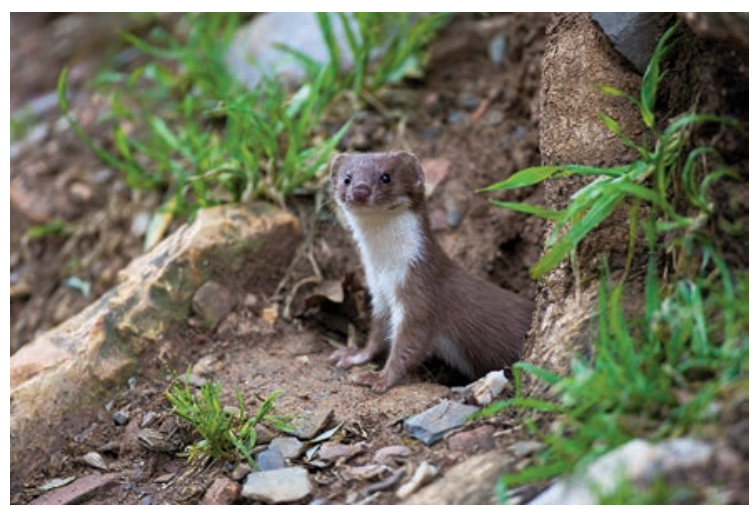

Figure 2. The weasel, Mustela nivalis Linnaeus, 1766, is the sole carnivore which occurs on Asinara (photo by Riccardo Romanelli). 
NATO radiogoniometric station was constructed on the eastern half of the island. As a result of this, the animals escaped from their guardians' control giving origin to a new population, the descendants of which have survived up to the present. According to Ruiu \& Trainito (1999), Tavolara represents the territory in which the Sardinian subadults of golden eagle, Aquila chrysaetos (Linnaeus, 1758), coming in particular from the hills of Gallura and Monte Nieddu, spend their first winter to practice hunting techniques: "They mostly look for goats. First hair kids, to claw on the fly to avoid the furious defense of adult" individuals of the new population of feral goats today available on the small island. According to Couturier (1959), the original wild goats of Tavolara were instead the same as the goats of the northern Tyrrhenian island of Montecristo. They were characterised by morphological patterns which fall within the phenotype of Capra aegagrus dorcas Reichenow, 1888, typical of the island of Youra, in the Northern Sporades archipelago (Western Aegean Sea, Greece) (Masseti, 2009). A few original wild goats of Tavolara were collected before their extinction in the wild, and were bred in a free-ranging state until recently in the Ogliastra bush, within the perimeter of the Gulf of Orosei and Gennargentu National Park, where they returned to the wild after their abandonment by the shepherds (Fig. 4). They survived in the new territories of distribution at least up to the end of the first decade of 2000s. It seems that these goats were brought from Tavolara over 120 years ago, in the second half of the 800 s.

Mouflons, Ovis orientalis (Gmelin, 1774), are only present on Asinara (Torre \& Monbailliu, 1993; Massoli Novelli, 2003), and Figarolo (Pratesi \& Tassi, 1973; Ruiu, 1993; Bocchieri \& Satta, 1999; Trainito, 2008; Trainito \& Navone, 2011) (Fig. 5). The stock of Asinara was introduced from the Natural Reserve of Capo Figari and the islet of Figarolo in 1952. Wild boars, Sus scrofa Linnaeus, 1758, occur permanently only on five islands: Asinara (Torre \& Monbailliu, 1993; Pedrotti \& Toso, 1999, 2002; Massoli Novelli, 2003), Spargi (Racheli; Pedrotti \& Toso, 1999, 2002), Caprera (Pedrotti \& Toso, 1999, 2002), Maddalena (Pedrotti \& Toso, 2002) and Sant'Antioco, but they have been also reported swimming from Asinara to the near Isola Piana (Bazzoni, 2013). Modern scientific investigation considers wild boars as quite

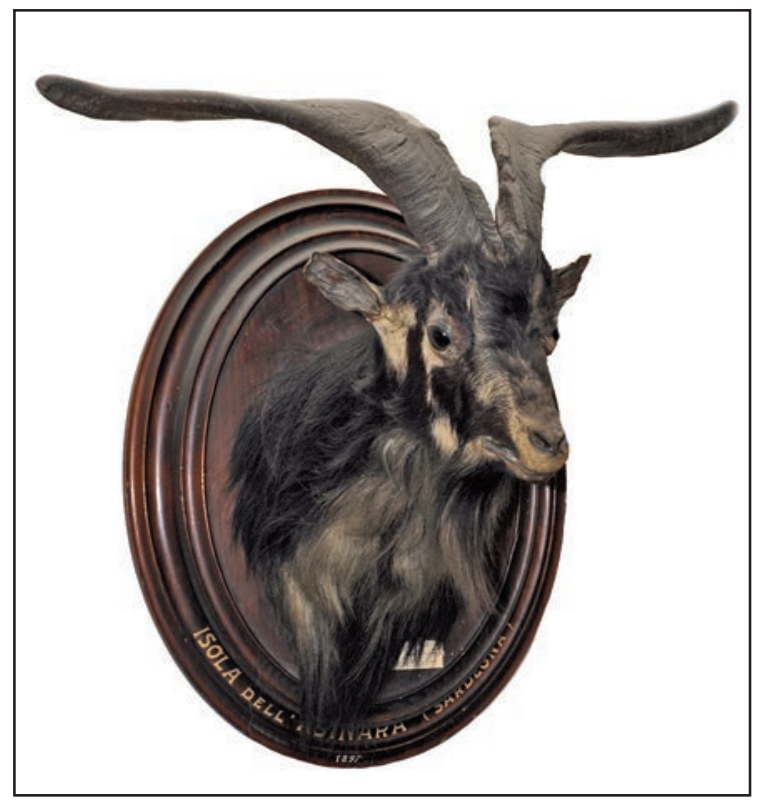

Figure 3. Trophy of an adult male captured on the small island of Asinara in 1897, and preserved in the collection of the Museum of Natural History of the University of Florence Zoological Section (MZUF no. coll. 11943) (photo by Saulo Bambi, courtesy Zoological Museum "La Specola" of the University of Florence).

competent swimmers, although they cannot survive a crossing of more than a few miles of open sea. In fact, they have been often reported swimming across the narrow marine straits separating the islets from the continental shores of the Mediterranean (Masseti, 2007, 2012). All the wild boars have been introduced on the satellite islands of Sardinia for hunting purposes in rather recent times. Regarding the ungulate domestic breeds, Sardinia has always been considered the stronghold of donkeys. Nowadays, however, these equids are no longer as widespread as they were in the past. In a still recent past, they demonstrated a substantial variation in size, ranging from normal dimensions to those typical of the Mediterranean dwarf varieties (cf. Masseti, 2012), like the donkeys that are still reared on the island of Asinara (see Pinna et al., 1990; Pinna et al., 1993; Cossu et al., 1994) (Fig. 6).

Data on the distribution of chiropters on the satellite islands of Sardinia have been provided by Zava \& Violani (1992), Grafitti \& Mucedda (1995), Zava et al. (1995), Fornasari et al. (1997), Mocci Demartis \& Secci (1997), Skiba (2009), Winter et 
al. (2015). Very recently, Mucedda et al. (2016) published an important overview of the bats of $\mathrm{La}$ Maddalena, Caprera, Santo Stefano, Spargi, Budelli, Santa Maria, Tavolara, Molara, Figarolo, Asinara, Isola Piana (off Asinara), San Pietro, Sant'Antioco, Serpentara and Isola dei Cavoli. Of the 21 species of bats found in Sardinia, at least 11 were recorded from the satellite islands. The species most widespread is the common pipistrelle, Pipistrellus pipistrellus (Schreber, 1774), which is present on 15 islands, followed by the European free-tailed bat, Tadarida teniotis (Rafinesque, 1814) (Fig. 7), on 12 islands. The Savi's pipistrelle, Hypsugo savii (Bonaparte, 1837), and the Kuhl's pipistrelle, Pipistrellus kuhlii (Kuhl, 1817) are instead reported from nine islands. The rarest species is the

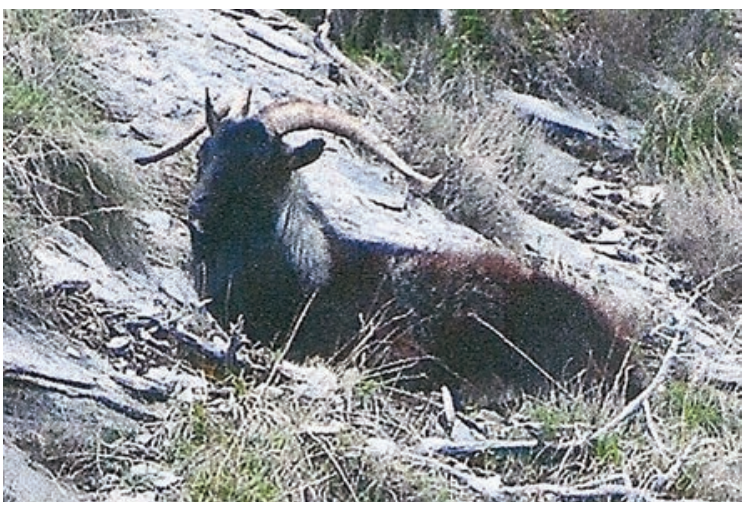

Figure 4. Original wild goats of Tavolara, before their extinction in the wild, were bred in a free-ranging state until recently in the Ogliastra bush, Gulf of Orosei and Gennargentu NP.

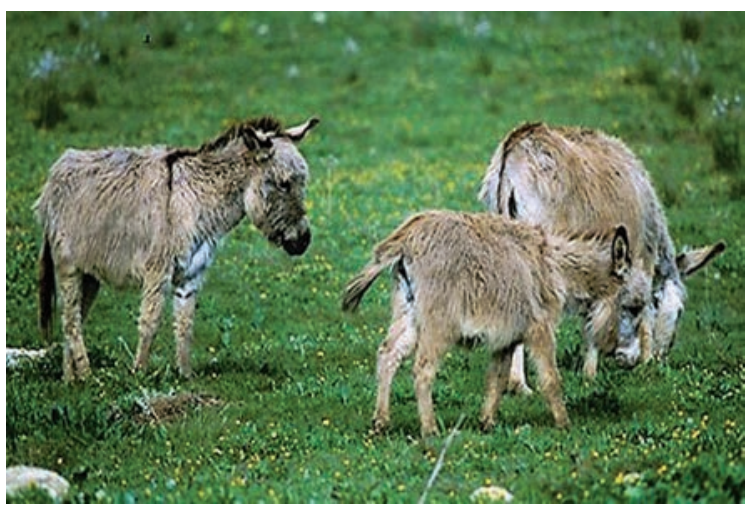

Figure 6. A small race of donkey is still bred on the island of Asinara, off the northwestern coast of Sardinia (photo by Marco Masseti).
Daubenton's bat, Myotis daubentonii (Kuhl, 1817), which has been observed only on Asinara. No individual of the endemic Sardinian long-eared bat, Plecotus sardus Mucedda, Kiefer, Pidinchedda and Veith, 2001, has been recorded from any of the islets, being its distribution limited to a few wooded areas of central and central-eastern Sardinia. Mucedda et al. (2016) are also of the opinion that the lesser mouse-eared bat, Myothis blythi (Tomes, 1857), has been erroneously reported from San Pietro by Zava \& Violani (1992), since the only two species of the genus Myotis occurring in Sardinia are the long-fingered bat, $M$. capaccini (Bonaparte, 1837) - reported by Capizzi \& Santini (1999d, 2002d) from La Maddalena, and Mucedda et al. (2016) from San Pietro - and the Maghreb

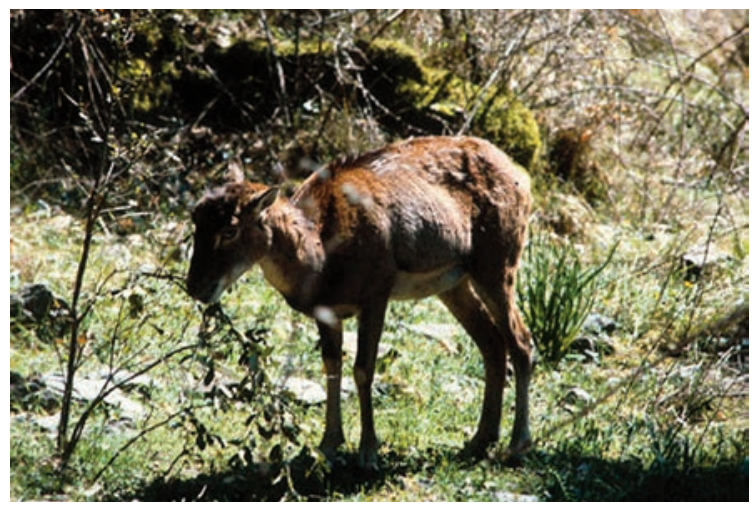

Figure 5. Introduced populations of mouflons, Ovis orientalis (Gmelin, 1774), are present today on Asinara and Figarolo (photo by Marco Masseti).

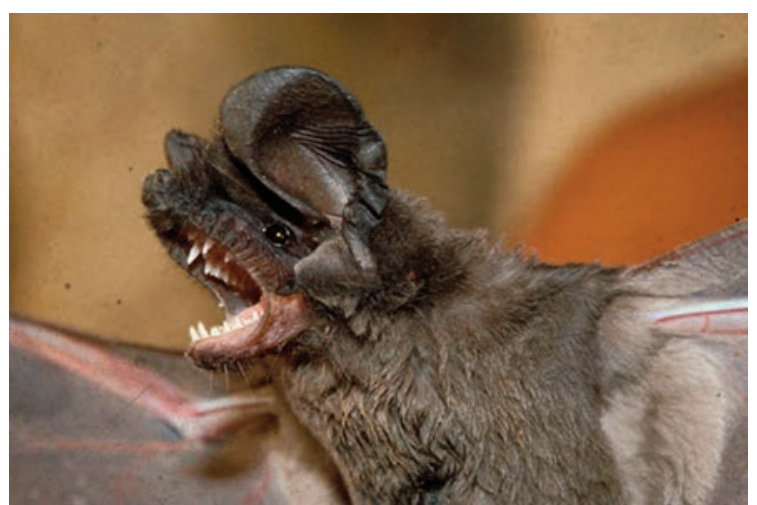

Figure 7. Portrait of an adult European free-tailed bat, Tadarida teniotis (Rafinesque, 1814) (photo by Bruno Zava). 


\begin{tabular}{|c|c|c|}
\hline island & species & references \\
\hline Razzoli & Oryctolagus cuniculus ${ }^{1}$; Rattus rattus ${ }^{2-3}$ & $\begin{array}{l}{ }^{1} \text { Spagnesi, 1999b, 2002b; }{ }^{2} \text { Capizzi \& Santini, 1999d, 2002d; } \\
{ }^{3} \text { Martin et al., 2000; }\end{array}$ \\
\hline Budelli & $\begin{array}{l}\text { Pipistrellus pipistrellus }{ }^{1,3} \text {; Tadarida teniotis }{ }^{3} \text {; } \\
\text { Rattus rattus }{ }^{2} \text {; }\end{array}$ & $\begin{array}{l}\text { 'Mucedda et al., 2015; }{ }^{2} \text { Martin et al., 2000; }{ }^{3} \text { Mucedda et al., } \\
\text { 2015; }\end{array}$ \\
\hline Spargiotto & Oryctolagus cumiculus ${ }^{1}$; & ${ }^{1}$ Spagnesi, 1999b, 2002b; \\
\hline Spargi & 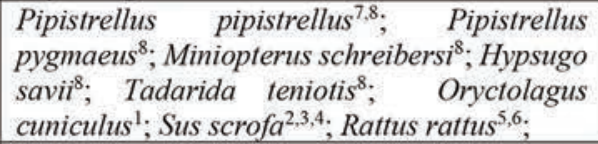 & $\begin{array}{l}{ }^{1} \text { Spagnesi, 1999b, 2002b; }{ }^{2} \text { Racheli; }{ }^{3} \text { Pedrotti \& Toso, } 1999 ; \\
{ }^{4} \text { Pedrotti \& Tosso, 2002; }{ }^{5} \text { Capizzi \& Santini, 1999d, 2002d; } \\
{ }^{6} \text { Martin et al., 2000; }{ }^{7} \text { Mucedda et al., 2015; }{ }^{8} \text { Mucedda et al., } \\
2015\end{array}$ \\
\hline Santa Maria & $\begin{array}{l}\begin{array}{l}\text { Oryctolagus cumiculus }{ }^{1} ; \\
\text { pipistrellus }^{2,4}, \text { Rattus rattus }^{3} ;\end{array}\end{array}$ & $\begin{array}{l}\text { 'Spagnesi, 1999b, 2002b; }{ }^{2} \text { Mucedda et al., 2015; }{ }^{3} \text { Martin et al., } \\
\text { 2000; }{ }^{4} \text { Mucedda et al., 2016; }\end{array}$ \\
\hline La Maddalena & 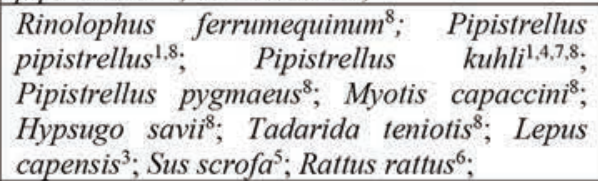 & $\begin{array}{l}{ }^{1} \text { Mucedda et al., 2015; }{ }^{2} \mathrm{CK} \text { map recorded in } 1987 ;{ }^{3} \text { Spagnesi, } \\
\text { 1999a, 2002a; }{ }^{4} \mathrm{MZUF}-13036 ;{ }^{5} \text { Pedrotti \& Tosso, 2002; } \\
{ }^{6} \mathrm{Capizzi} \text { \& Santini, 1999d, 2002d; 'Zava et al. (1996); } \\
{ }^{8} \text { Mucedda et al., 2016; }\end{array}$ \\
\hline Santo Stefano & 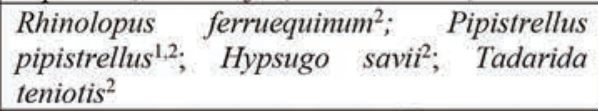 & ${ }^{1}$ Mucedda et al., 2015; ${ }^{2}$ Mucedda et al., 2015; \\
\hline Caprera & 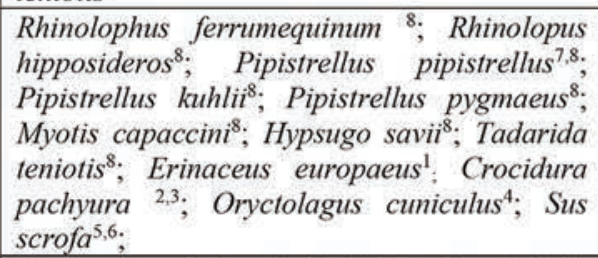 & $\begin{array}{l}{ }^{1} \text { Cossu et al., 1994; }{ }^{2} \text { Amori \& Masseti, 1996; }{ }^{3} \text { Thibault et al., } \\
\text { 1988: }{ }^{4} \text { Spagnesi, 1999b, } 2002 \text { b; }{ }^{5} \text { Pedrotti \& Toso, 1999; } \\
{ }^{6} \text { Pedrotti \& Tosso, 2002; }{ }^{7} \text { Mucedda et al., 2015; }{ }^{8} \text { Mucedda et } \\
\text { al., 2016; }\end{array}$ \\
\hline Figarolo & $\begin{array}{l}\text { Pipistrellus pipistrellus }{ }^{1,7} ; \text { Pipistrellus } \text { kuhlii }^{7} \text {; } \\
\text { Hypsugo savii }{ }^{7} ; \text { Tadarida teniotis }{ }^{7} ; \quad \text { Ovis } \\
\text { orientalis }^{2,3,4,5,6} \text {; }\end{array}$ & $\begin{array}{l}{ }^{1} \text { Mucedda } \text { et al., 2015; }{ }^{2} \text { Pratesi \& Tassi, } 1973 ;{ }^{3} \text { Ruiu, } 1993 ; \\
{ }^{4} \text { Bocchieri \& Satta, } 1999 ;{ }^{3} \text { Trainito, 2008; }{ }^{6} \text { Trainito \& Navone, } \\
\text { 2011; }{ }^{7} \text { Mucedda } \text { et al., 2016; }\end{array}$ \\
\hline Tavolara & 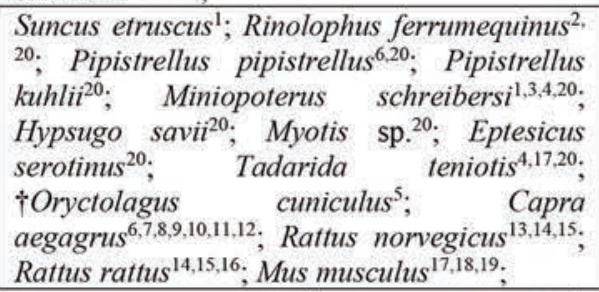 & 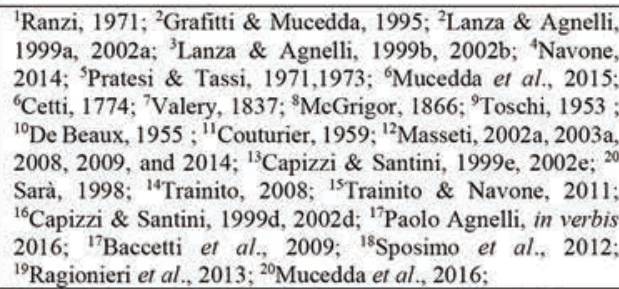 \\
\hline $\begin{array}{l}\text { Isola Piana off } \\
\text { Tavolara }\end{array}$ & $\begin{array}{l}\text { Pipistrellus pipistrellus }{ }^{1,4} \text {; Pipistrellus kuhlii } \\
\text { †Oryctolagus } \\
\text { muscuniculus }{ }^{2} \text {; Rattus rattus }{ }^{3} \text {; Mus }\end{array}$ & $\begin{array}{l}{ }^{1} \text { Mucedda et al., 2015; }{ }^{2} \text { Trainito, } 2008 ;{ }^{2} \text { Trainito \& Navone, } \\
\text { 2011; }{ }^{3} \text { Paolo Sposimo, in litteris } 2014 ;{ }^{4} \text { Mucedda et al., 2016; }\end{array}$ \\
\hline Isola dei Cavalli & Rattus rattus $^{1}$; Mus musculus ${ }^{1}$ & ${ }^{1}$ Paolo Sposimo, in litteris 2014; \\
\hline Proratora & Rattus rattus $^{1} ;$, Mus musculus ${ }^{1}$ & ${ }^{1}$ Paolo Sposimo, in litteris 2014; \\
\hline $\begin{array}{l}\text { Reuloino or } \\
\text { Isolotto Rosso }\end{array}$ & Mus musculus & 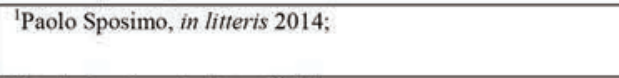 \\
\hline Molarotto & $\dagger$ Rattus rattus & ${ }^{3}$ Paolo Sposimo, in litteris 2014; \\
\hline Molara & 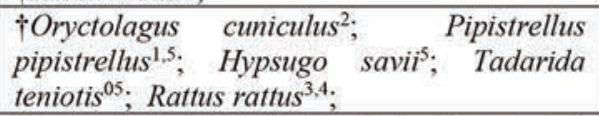 & 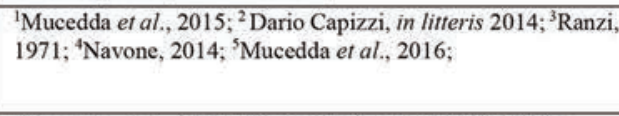 \\
\hline Serpentara & $\begin{array}{l}\text { Pipistrellus pipistrellus }{ }^{1,3} ; \text { Tadarida }^{\text {teniotis }}{ }^{3} \text {; } \\
\text { Rattus rattus }\end{array}$ & ${ }^{1}$ Mucedda et al,, 2015; ${ }^{2}$ Capizzi \& Santini, 1999d, 2002d; \\
\hline Isola dei Cavoli & $\begin{array}{l}\text { Pipistrellus pipistrellus }{ }^{1,3} \text {; Pipistrellus kuhlii } \\
\text { Vulpes }\end{array}$ & $\begin{array}{l}{ }^{1} \text { Mucedda et al., 2015; }{ }^{2} \text { Scrugli \& Cogoni, } 1995 ;{ }^{3} \text { Mucedda et } \\
\text { al., 2015; }\end{array}$ \\
\hline Isola Rossa & Oryctolagus cumiculus ${ }^{1}$; & ${ }^{1}$ Spagnesi, 1999b, 2002b; \\
\hline Vacca & 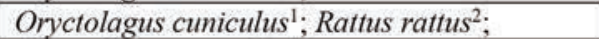 & ${ }^{1}$ Spagnesi, 1999b, 2002b; 2Capizzi \& Santini, 1999d, 2002d; \\
\hline Toro & Oryctolagus cuniculus ${ }^{1}$ & ${ }^{1}$ Spagnesi, 1999b, 2002b; \\
\hline
\end{tabular}

Table 1/1. Terrestrial mammals of the satellite islands of Sardinia. MZUF: Museo di Zoologia "La Specola" dell’Università di Firenze; MCSNM: Museo Civico di Storia naturale di Milano. 


\begin{tabular}{|c|c|c|}
\hline island & species & references \\
\hline Sant'Antioco & $\begin{array}{l}\text { Pipistrellus pipistrellus }{ }^{1} \text {; Myotis punicus }{ }^{2,3,4.5} \text {; } \\
\text { Sus scrofa }{ }^{6 \mathrm{C}} \text {; }\end{array}$ & $\begin{array}{l}{ }^{1} \text { Mucedda et al., 2015; }{ }^{2} \text { MSNM } 722 ;{ }^{3} \text { Zava \& Violani (1992); } \\
{ }^{4} \text { Mucedda et al. 2016; }{ }^{5} \text { Zava et al. (1996); }{ }^{6} \text { present work; }\end{array}$ \\
\hline $\begin{array}{l}\text { Isolotto di Cala } \\
\text { Vinagra }\end{array}$ & Tadarida teniotis $^{1}$ & ${ }^{1}$ Fornasari et al, 1997; \\
\hline San Pietro & 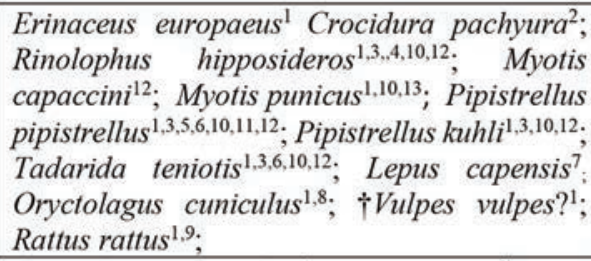 & 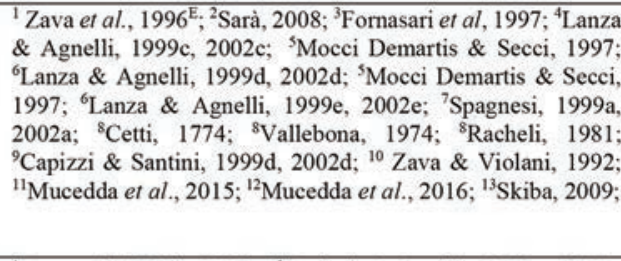 \\
\hline Mal di Ventre & $\begin{array}{l}\text { Oryctolagus cuniculus }{ }^{1} \text {; Rattus } \text { rattus }^{2} ; \text { Mus } \\
\text { musculus }{ }^{2,3} \text {; }\end{array}$ & $\begin{array}{l}{ }^{1} \text { Spagnesi, 1999b, 2002b; }{ }^{2} \text { Capizzi \& Santini, 1999c, 2002c; } \\
{ }^{3} \text { Andreotti et al., 2001; }\end{array}$ \\
\hline \multicolumn{3}{|l|}{ Foradada } \\
\hline $\begin{array}{l}\text { Isola Piana, off } \\
\text { Asinara }\end{array}$ & Rattus rattus ${ }^{1} ;$ Sus scrof $\mathrm{a}^{2}$; & ${ }^{1}$ Martin et al., 2000; ${ }^{2}$ Bazzoni, 2013; \\
\hline Asinara & 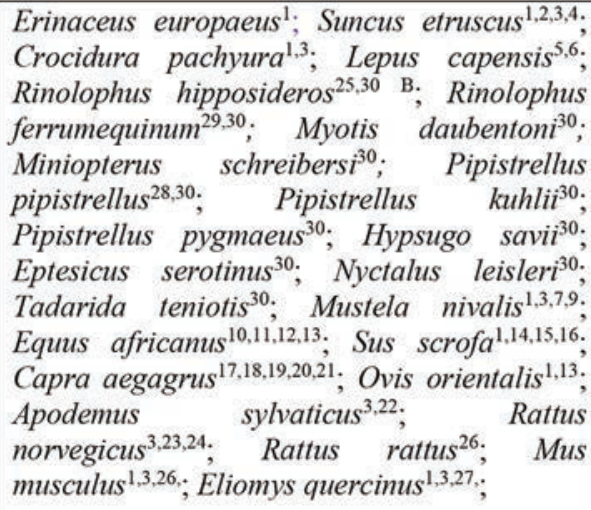 & 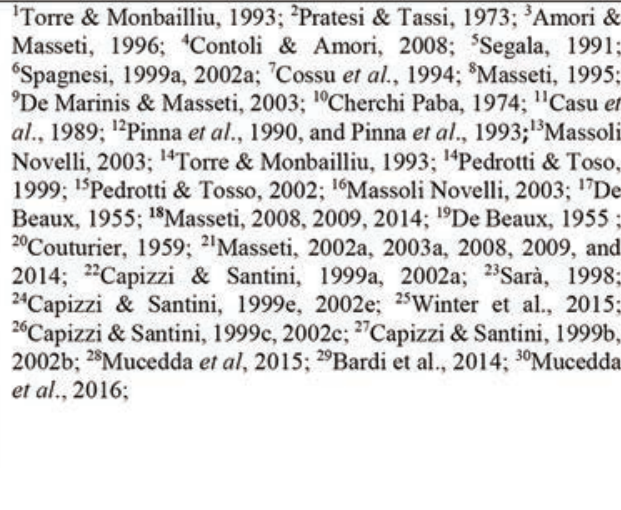 \\
\hline
\end{tabular}

Table 1/2. Terrestrial mammals of the satellite islands of Sardinia. MZUF: Museo di Zoologia "La Specola" dell'Università di Firenze; MCSNM: Museo Civico di Storia naturale di Milano.

mouse-eared bat, M. punicus Felten, Spitzenberger et Storch, 1977, recorded by Skiba (2009) from Sant'Antioco.

\section{CONCLUSIONS}

According to the data collected in the present study the terrestrial mammalian fauna of the circum-Sardinian islands amounts to 28 species, including 13 bats (Rhinolophus ferrumequinum, Rhinolopus hipposideros. Myotis capaccini. Myotis daubentoni, Myotis punicus, Pipistrellus pipistrellus, Pipistrellus kuhli, Pipistrellus pygmaeus, Miniopterus schreibersi, Hypsugo savii, Eptesicus serotinus, Nyctalus leisleri, and Tadarida teniotis) and 15 non-volant mammals (Erinaceus europaeus, Crocidura pachyura, Suncus etruscus, Lepus capensis, Oryctolagus cuniculus, Vulpes vulpes, Mustela nivalis, Sus scrofa, Capra aegagrus, Ovis orientalis, Apodemus sylvaticus, Rattus norvegicus, Rattus rattus, Mus musculus, and Eliomys querci$n u s$ ) (Table 1). Today, a part from the bats, the faunistic horizons of all these islands are no more characterised by endemic Pleistocene species. The exclusive present occurrence of non-volant continental mammals on the satellite islands of Sardinia seems to be linked essentially to the introduction by man during the Holocene.

\section{ACKNOWLEDGEMENTS}

Among the many friends and colleagues who helped me as I was preparing this paper, I would like to express my appreciation and gratitude in par- 
ticular to Paolo Agnelli, Museo di Zoologia "La Specola" dell'Università di Firenze (Italy); Dario Capizzi, Direzione Regionale Ambiente e Sistemi Naturali, Regione Lazio (Italy); Paolo Sposimo, NEMO Nature Environment Management Operators, Firenze (Italy), and Bruno Zava, Wilderness Studi Ambientali, Palermo (Italy). Special thanks are due to Mauro Mucedda, Gruppo Italiano Ricerca Chirotteri (Sassari, Italy), for having set at my disposal the results of the study carried out on the bats of the small islands of Sardinia.

\section{REFERENCES}

Amori G. \& Masseti M., 1996. Does the occurrence of predators on Central Mediterranean islands affect the body size of micromammals? Vie et Mileau, 46: 205211.

Andreotti A., Baccetti N., Perfetti A., Besa M., Genovesi P. \& Guberti V., 2001. Mammiferi ed Uccelli esotici in Italia: analisi del fenomeno, impatto sulla biodiversità e linee guida gestionali. Quaderni di Conservazione della Natura, 2, Ministero dell'Ambiente. Istituto Nazionale Fauna Selvatica, Ozzano dell'Emilia (Bologna), 192 pp.

Baccetti N., Capizzi D., Corbi F., Massa B., Nissardi S., Spano G. \& Spisomo P., 2009. Breeding shearwaters on Italian islands: population size, island selection and co-existence with their main alien predator, the black rat. Rivista italiana d'Ornitologia, 78: 83-100.

Bardi A., Benassi G., Fornari A.R., Panchetti F. \& Sanna S., 2014. Piano di gestione del SIC "Itb010082 Isola dell'Asinara”. Repubblica Italiana, Regione Autonoma della Sardegna, Cagliari, 238 pp.

Bazzoni G., 2013. Isola Piana devastata dai cinghiali. La Nuova Sardegna, edizione Sassari: 8 settembre 2013.

Bocchieri E. \& Satta V., 1999. Flora and vegetal landscape on the island of Figarolo (NE Sardinia). Lagascalia, 21: 17- 46.

Capizzi D. \& Santini L., 1999a. Topo selvatico Apodemus sylvaticus (Linnaeus, 1758). In: Spagnesi M. \& Toso S. (Eds.), Iconografia dei Mammiferi d'Italia. Istituto Nazionale Fauna Selvatica "Alessandro Ghigi”. Ozzano dell'Emilia (Bologna), pp. 131-132.

Capizzi D. \& Santini L., 2002a. Topo selvatico Apodemus sylvaticus (Linnaeus, 1758). In: Spagnesi M. \& De Marinis A.M. (Eds.), Mammiferi d'Italia. Quaderni di Conservazione della Natura, 14. Ministero dell' Ambiente - Istituto Nazionale Fauna Selvatica. Ozzano dell'Emilia (Bologna), pp. 199-200.

Capizzi D. \& Santini L., 1999b. Quercino Eliomys quercinus (Linnaeus, 1766). In: Spagnesi M. \& Toso S. (Eds.), Iconografia dei Mammiferi d'Italia. Istituto
Nazionale Fauna Selvatica “Alessandro Ghigi”. Ozzano dell'Emilia (Bologna), p. 114.

Capizzi D. \& Santini L., 2002b. Quercino Eliomys quercinus (Linnaeus, 1766). In: Spagnesi M. \& De Marinis A.M. (Eds.), Mammiferi d'Italia. Quaderni di Conservazione della Natura, 14. Ministero dell' Ambiente - Istituto Nazionale Fauna Selvatica. Ozzano dell'Emilia (Bologna), pp. 68-169.

Capizzi D. \& Santini L., 1999c. Topo domestico Mus domesticus Schwarz et Schwarz, 1943. In: Spagnesi M. \& Toso S. (Eds.), Iconografia dei Mammiferi d'Italia. Istituto Nazionale Fauna Selvatica "Alessandro Ghigi”. Ozzano dell'Emilia (Bologna), p. 134.

Capizzi D. \& Santini L., 2002c. Topo domestico Mus domesticus Schwarz et Schwarz, 1943. In: Spagnesi M. \& De Marinis A.M. (Eds.), Mammiferi d'Italia. Quaderni di Conservazione della Natura, 14. Ministero dell' Ambiente - Istituto Nazionale Fauna Selvatica. Ozzano dell'Emilia (Bologna), pp. 203204.

Capizzi D. \& Santini L., 1999d. Ratto nero o dei tetti Rattus rattus (Linnaeus, 1758). In: Spagnesi M. \& Toso S. (Eds.), Iconografia dei Mammiferi d'Italia. Istituto Nazionale Fauna Selvatica "Alessandro Ghigi”. Ozzano dell'Emilia (Bologna), pp. 135-136.

Capizzi D. \& Santini L., 2002d . Ratto nero o dei tetti Rattus rattus (Linnaeus, 1758). In: Spagnesi M. \& De Marinis A.M. (Eds.), Mammiferi d'Italia. Quaderni di Conservazione della Natura, 14. Ministero del1'Ambiente - Istituto Nazionale Fauna Selvatica. Ozzano dell'Emilia (Bologna), pp. 205-206.

Capizzi D. \& Santini L., 1999e. Ratto delle chiaviche Rattus norvegicus (Berkenhout, 1769). In: Spagnesi M. \& Toso S. (Eds.), Iconografia dei Mammiferi d'Italia. Istituto Nazionale Fauna Selvatica "Alessandro Ghigi”. Ozzano dell'Emilia (Bologna), p. 137.

Capizzi D. \& Santini L., 2002e. Ratto delle chiaviche Rattus norvegicus (Berkenhout, 1769). In: Spagnesi M. \& De Marinis A.M. (Eds.), Mammiferi d'Italia. Quaderni di Conservazione della Natura, 14. Ministero dell' Ambiente - Istituto Nazionale Fauna Selvatica. Ozzano dell'Emilia (Bologna), pp. 207-208.

Carbone F., 1998. Coste e piccole isole. Editrice Dattena, Cagliari, 48 pp.

Casu T., Lai G. \& Pinna G., 1989. Guida alla flora e alla fauna della Sardegna. Editrice Archivio Fotografico Sardo, Nuoro, 417 pp.

Cetti F., 1774. Storia naturale dei Quadrupedi della Sardegna. G. Piattoli, Sassari, 452 pp.

Contoli L. \& Amori G., 2008. Suncus etruscus (Savi, 1822). In: Amori G., Contoli L. \& Nappi A. (Eds.), Fauna d'Italia. Mammalia II. Erinaceomorpha, Soricomorpha, Lagomorpha, Rodentia. Edizione Calderini del Il Sole 24 ORE, Business media, Milano, pp. 179-183. 
Cherchi Paba F., 1974. Evoluzione storica dell'attività industriale agricola caccia e pesca in Sardegna. Vol. I-VI. Regione Autonoma Sarda, Cagliari, 452 pp.

CK map/Checklist and Distribution of the Italian fauna,http./www.moinambiente.it/index.php?id_sexione $=1930$.

Cossu A., Monballiu X. \& Torre A., 1994. L'isola dell'Asinara. Delfino, Sassari, 79 pp.

De Marinis A.M. \& Masseti M., 2003. The weasel (Mustela nivalis) on the Mediterranean islands. Mammalian Biology, 68: 181-186. https://doi.org/10. 1078/1616-5047-00081

Fornasari L., Violani C. \& Zava B., 1997. I chirotteri italiani. L'Epos, Palermo, 132 pp.

Grafitti G. \& Mucedda M., 1995. Le grotte dell'isola di Tavolara e la loro fauna. Biogeographia, 18: 51-62.

Kiefer A. \& Veith M., 2001. A new species of long-eared bat from Europe (Chiroptera: Vespertilionidae). Myotis, 39: 5-16.

Kiefer A., Kosuch J. Veith M., Mayer F. \& Helversen O., von, 2002. Conflicting molecular phylogenies of European long-eared bats (Plecotus) can be explained by cryptic diversity. Molecular Phylogenetics and Evolution, 25: 557-566. https://doi.org/10.1016/ S1055-7903(02)00299-3

Lanza B. \& Agnelli P., 1999a. Rinolofo maggiore Rinolophus ferrumequinum (Schreber, 1774). In: Spagnesi M. \& Toso S. (Eds.), Iconografia dei Mammiferi d'Italia. Istituto Nazionale Fauna Selvatica "Alessandro Ghigi”. Ozzano dell'Emilia (Bologna), pp. 33-34.

Lanza B. \& Agnelli P., 1999b. Miniottero di Schreiber Miniopterus schreibersi (Kuhl, 1817). In: Spagnesi M. \& Toso S. (Eds.), Iconografia dei Mammiferi d'Italia. Istituto Nazionale Fauna Selvatica "Alessandro Ghigi”. Ozzano dell'Emilia (Bologna), pp. 92-94.

Lanza B. \& Agnelli P., 1999c. Rinolofo minore Rhinolophus hipposideros (Bechstein, 1800). In: Spagnesi M. \& Toso S. (Eds.), Iconografia dei Mammiferi d'Italia. Istituto Nazionale Fauna Selvatica "Alessandro Ghigi”. Ozzano dell'Emilia (Bologna), pp. 35-36.

Lanza B. \& Agnelli P., 1999d. Pipistrello nano, Pipistrellus pipistrellus (Schreber, 1774). In: Spagnesi M. \& Toso S. (Eds.), Iconografia dei mammiferi d'Italia. Istituto Nazionale Fauna Selvatica "Alessandro Ghigi”. Ozzano dell'Emilia (Bologna), pp. 83-85.

Lanza B. \& Agnelli P., 1999e. Molosso del Cestoni Tadarida teniotis (Rafinesque, 1814). In: Spagnesi M. \& Toso S. (Eds.), Iconografia dei Mammiferi d'Italia. Istituto Nazionale Fauna Selvatica "Alessandro Ghigi”. Ozzano dell'Emilia (Bologna), pp. 95-96.

Lanza B. \& Agnelli P., 2002a. Rinolofo maggiore Rinolophus ferrumequinum (Schreber, 1774). In: Spagnesi M. \& De Marinis A.M. (Eds.), Mammiferi d'Italia. Quaderni di Conservazione della Natura, 14. Ministero dell' Ambiente - Istituto Nazionale Fauna Sel- vatica. Ozzano dell'Emilia (Bologna), pp. 52-54.

Lanza B. \& Agnelli P., 2002b. Miniottero di Schreiber Miniopterus schreibersi (Kuhl, 1817). In: Spagnesi M. \& De Marinis A.M. (Eds.), Mammiferi d'Italia. Quaderni di Conservazione della Natura, 14. Ministero dell' Ambiente - Istituto Nazionale Fauna Selvatica. Ozzano dell'Emilia (Bologna), pp. 136-139.

Lanza B. \& Agnelli P., 2002c. Rinolofo minore Rhinolophus hipposideros (Bechstein, 1800). In: Spagnesi M. \& De Marinis A.M. (Eds.), Mammiferi d'Italia. Quaderni di Conservazione della Natura, 14. Ministero dell' Ambiente - Istituto Nazionale Fauna Selvatica. Ozzano dell'Emilia (Bologna), pp. 55-56.

Lanza B. \& Agnelli P., 2002d. Pipistrello nano, Pipistrellus pipistrellus (Schreber, 1774). In: Spagnesi M. \& De Marinis A.M. (Eds.), Mammiferi d'Italia. Quaderni di Conservazione della Natura, 14. Ministero dell' Ambiente - Istituto Nazionale Fauna Selvatica. Ozzano dell'Emilia (Bologna), pp. 122125.

Lanza B. \& Agnelli P., 2002e. Molosso del Cestoni Tadarida teniotis (Rafinesque, 1814). In: Spagnesi M. \& De Marinis A.M. (Eds.), Mammiferi d'Italia. Quaderni di Conservazione della Natura, 14. Ministero dell' Ambiente - Istituto Nazionale Fauna Selvatica. Ozzano dell'Emilia (Bologna), pp. 140-142.

Martin J.L., Thibault J.C. \& Bretagnolle V., 2000 Black rats, island characteristics, and colonial nesting birds in the mediterranean: consequences of an ancient introduction. Conservation Biology, 14: 1452-1466.

Massari S., 2013. Un viaggio in Sardegna. Youcanprint, 116 pp.

Masseti M., 1995. Quaternary biogeography of the Mustelidae family in the Mediterranean islands. Hystrix (n.s.), 7: 17-34.

Masseti M., 2007. The economic role of Sus in early human fishing communities. In: Albarella U., Dobney K., Ervynck A. \& Rowley-Conwey P. (Eds.), Pigs and humans 10,000 years of interaction. Oxford University Press, Oxford/New York, pp. 156-170.

Masseti M., 2008. Origin and artificial distribution of the wild goat, Capra aegagrus Erxleben, 1777, on the islands of the Mediterranean Sea and the Eastern Atlantic Ocean. In: Granados Teorres J.E., CanoManuel León J., Fandos Paris P. \& Cadenas de Llano Aguilar R. (a cura di), Tendencias actuales en el Estudio y Conservacion de los Caprinos Europeos. II Congreso Internacional del género Capra en Europa (Granada, 20-23 novembre 2007). Junta de Andalucia, Granada, pp. 169-195.

Masseti M., 2009. The wild goats, Capra aegagrus Erxleben, 1777, of the Mediterranean Sea and the Eastern Atlantic Ocean islands. Mammal Review, 39: 141-157. https://doi.org/10.1111/j.1365-2907.2009. 00141.x 
Masseti M., 2012. Atlas of terrestrial mammals of the Ionian and Aegean islands. De Gruyter, Berlin, 302 pp.

Masseti M., 2014. Las cabras salvajes, 'Capra aegagrus' Erxleben, 1777, de las islas del Mediterráneo/The wild goats, "Capra aegagru” Erxleben, 1777, of the Mediterranean islands. In: Seguí B. (ed.), Balearean Boc. Four millennia of history, ten years of homologation. Consell de Mallorca. Palma de Mallorca, pp. 94-107.

Massoli Novelli R., 2003. L'isola dei mufloni. Il Parco Regionale dell'Asinara. Diana, 2: 32-35.

Mocci Demartis A. \& Secci A., 1997. Dati sulla distribuzione dei Chirotteri nella Sardegna meridionale. Rendiconti Seminario Facoltà Scienze, Università di Cagliari, 67: 61-74.

Mucedda M., Kiefer A., Pidinchedda E. \& Veith M., 2002. A new species of long-eared bat (Chiroptera, Vespertilionidae) from Sardinia. Acta Chiropterologica, 42: 121-135.

Mucedda M., Pidinchedda E. \& Bertelli M.L., 2015 Note sui pipistrelli nelle piccole isole della Sardegna. In: Mucedda M., Roscioni F. \& Preatoni D.G. (Eds.), Riassunti: Comunicazioni e Poster del III Convegno Italiano sui Chirotteri, Trento, 9-11 ottobre 2015, p. 22.

Mucedda M., Pidinchedda E. \& Bertelli M.L., 2016. Note sui pipistrelli nelle piccole isole della Sardegna. In: Mucedda M., Roscioni F. \& Preatoni D.G. (Eds.), III Convegno Italiano sui Chirotteri, Trento, 9-11 ottobre 2015. Gruppo Italiano Ricerca Chirotteri - Associazione Teriologica Italiana, pp. 20-25.

Navone A., 2014. Piano di gestione del SIC Isola di Tavolara, Molara e Molarotto (ITB 010010). Area Marina Protetta Tavolara-Punta coda cavallo/Ministero dell' Ambiente e della Tutela del Territorio e del Mare, Roma, $251 \mathrm{pp}$,

Pedrotti L. \& Toso S., 1999. Cinghiale Sus scrofa Linnaeus, 1758. In: Spagnesi M. \& Toso S. (Eds.), Iconografia dei Mammiferi d'Italia. Istituto Nazionale Fauna Selvatica "Alessandro Ghigi”. Ozzano dell'Emilia (Bologna), pp. 166-167.

Pedrotti L. \& Toso S., 1999. Cinghiale Sus scrofa Linnaeus, 1758. In: Spagnesi M. \& De Marinis A.M., (Eds.), Mammiferi d'Italia. Quaderni di Conservazione della Natura, 14. Ministero dell'Ambiente Istituto Nazionale Fauna Selvatica. Ozzano dell'Emilia (Bologna), pp. 249-252

Pinna W., Vacca G.M. \& Lai P., 1990. Rilievi etno-demografici sull' asinello bianco dell' Asinara. Atti della Società Italiana di Scienze Veterinarie, 44: 17631767.

Pinna W., Cappio Borlino A., Vacca G.M. \& Lai P., 1993. Morphology of adult white donkeys of Asinara. Bollettino della Società Italiana di Biologia Sperimentale, 10: 595-600
Pratesi F. \& Tassi F., 1971. Guida alla natura d'Italia. Arnoldo Mondadori editore, Milano, 552 pp.

Pratesi F. \& Tassi F., 1973. Guida alla natura della Sardegna. Arnoldo Mondadori editore, Milano, 340 pp.

Racheli G., 1981. L'arcipelago del Sulcis e la sua storia. Vert Sardegna, Calasetta, Cagliari, 304 pp.

'Ragionieri L., Cutuli G., Sposimo P., Spano G., Navone A., Capizzi D., Baccetti N., Vannini \& Fratini S., 2013. Establishing the eradication unit of Molara Island: a case of study from Sardinia, Italy. Biological Invasions, 15: 2731-2742. https://doi.org/10.1007/ s10530-013-0487-y

Ranzi S., 1971. Ricerche zoologiche nelle isole di Tavolara e Molara. Quaderni de La Ricerca scientifica, 73: 25-28.

Ruiu D., 1993. Il muflone. Carlo Delfino editore, Sassari, $50 \mathrm{pp}$.

Ruiu D. \& Trainito E., 1999. Sapore di mare, sapore di montagna. Oasis, 1: 78-91.

Sarà M., 1998. I mammiferi delle isole del Mediterraneo. L'Epos, Palermo, 166 pp.

Sarà M., 2008. Crocidura pachyura (Küster, 1835). Descrizione morfologica. In: Amori G., Contoli L. \& Nappi A. (Eds.), Fauna d'Italia. Mammalia II. Erinaceomorpha, Soricomorpha, Lagomorpha, Rodentia. Edizione Calderini del Il Sole 24 ORE Business media, Milano, pp. 202-204.

Segala A., 1991. L'isola proibita. Oasis, 9: 28-47.

Scrugli A. \& Cogoni A., 1995. L'Isola dei cavoli. Carlo Delfino editore, Sassari, 45 pp.

Skiba R., 2009. Europäische Fledermäuse. Di neue Brehm Bücherei Bd. 648, Westarp Wissenschaften, Hohenwarsleben, 212 pp.

Spagnesi M., 1999a. Lepre sarda Lepus capensis Linnaeus, 1758. In: Spagnesi M. \& Toso S. (Eds.), Iconografia dei Mammiferi d'Italia. Istituto Nazionale Fauna Selvatica "Alessandro Ghigi". Ozzano dell'Emilia (Bologna), p. 104.

Spagnesi M., 2002a. Lepre sarda Lepus capensis Linnaeus, 1758. In: Spagnesi M. \& De Marinis A.M. (Eds.), Mammiferi d'Italia. Quaderni di Conservazione della Natura, 14. Ministero dell'Ambiente Istituto Nazionale Fauna Selvatica. Ozzano dell'Emilia (Bologna), pp. 152-153.

Spagnesi M., 1999b. Coniglio selvatico Oryctolagus cuniculus (Linnaeus, 1758). In: Spagnesi M. \& Toso S. (Eds.), Iconografia dei Mammiferi d'Italia. Istituto Nazionale Fauna Selvatica "Alessandro Ghigi”. Ozzano dell'Emilia (Bologna), pp. 98-99.

Spagnesi M., 2002b. Coniglio selvatico Oryctolagus cuniculus (Linnaeus, 1758). In: Spagnesi M. \& De Marinis A.M. (Eds.), Mammiferi d'Italia. Quaderni di Conservazione della Natura, 14. Ministero dell'Ambiente - Istituto Nazionale Fauna Selvatica. Ozzano dell'Emilia (Bologna), pp. 144-145. 
Sposimo P., Spano G., Navone A., Fratini S., Ragionieri L., Putzu M., Capizzi D. \& Baccetti N., 2012. Rodent eradication on Molara Island and surrounding islets (NE Sardinia): from success to the riddle of reinvasion. Aliens: The Invasive Species Bulletin, 32: 3338.

Thibault J.C., Guyot I., Martin J.L. \& Cheylan G., 1988. Observations sur les vertebrés terrestres des îles mineures de l'Archipel de la Maddalena. Travaux Scientifiques Parc Naturel Corse, 17: 47- 95.

Torre A. \& Monbailliu X., 1993. La Fauna. In: Cossu A., Gazale V., Monbailliu X. \& Torre A. (Eds.), Isole minori del Mediterraneo. Asinara. Storia, natura, mare e tutela ambientale. Edizioni Delfino, Sassari, 239 pp.

Trainito E., 2008. La fauna terrestre. In: Navone A. \& Trainito E. (Eds.), Tavolara. Carlo Delfino editore, Sassari, pp. 92-103.

Trainito E. \& Navone A., 2011. Tavolara, Punta Coda cavallo. Guida all'Area Marina Protetta. Carlo
Delfino editore, Sassari, $255 \mathrm{pp}$.

Vallebona G., 1974. Carloforte, storia di una colonizzazione (1738-1816). Arti Grafiche Bicidi, Genova, $144 \mathrm{pp}$.

Winter R., Treitler J., Kierdorf U., Schmidt S. \& Mantilla-Contreras J., 2015. Occurrence and activity patterns of bats in different habitat types in the northern part of the island of Asinara (Sardinia, Italy). In: Mucedda M., Roscioni F. \& Preatoni D.G. (Eds.), Riassunti: Comunicazioni e Poster del III Convegno Italiano sui Chirotteri, Trento, 9-11 ottobre 2015, p. 19

Zava B. \& Violani C., 1992, Nuovi dati sulla chirotterofauna italiana. Bollettino del Museo regionale di Scienze naturali, Torino, 10: 261- 264.

Zava B., Fiore M., Fornasari L. \& Violani C., 1995 Note sui Chirotteri dell'Isola di San Pietro con cenni storici sulle ricerche chirotterologiche in Sardegna. Biogeographia, 18: 641-651. 